\title{
Lack of Cas13a inhibition by anti-CRISPR proteins from Leptotrichia prophages
}

Alexander J Meeske ${ }^{1+}$, Matthew C. Johnson ${ }^{2}$, Logan T. Hille ${ }^{3-5}$, Benjamin P. Kleinstiver ${ }^{4-6}$, Joseph Bondy-Denomy ${ }^{2,7,8+}$

${ }^{1}$ Department of Microbiology, University of Washington, Seattle, WA 98109, USA

${ }^{2}$ Department of Microbiology and Immunology, University of California, San Francisco, San Francisco, CA 94158, USA

${ }^{3} \mathrm{PhD}$ Program in Biological and Biomedical Sciences, Harvard University, Boston, MA, 02115

${ }^{4}$ Center for Genomic Medicine, Massachusetts General Hospital, Boston, MA, 02114

${ }^{5}$ Department of Pathology, Massachusetts General Hospital, Boston, MA, 02114

${ }^{6}$ Department of Pathology, Harvard Medical School, Boston, MA, 02115

${ }^{7}$ Quantitative Biosciences Institute, University of California, San Francisco, San Francisco, CA 94158, USA

${ }^{8}$ Innovative Genomics Institute, Berkeley, CA 94720, USA

${ }^{+}$Co-corresponding authors

Meeske@uw.edu

Joseph.Bondy-Denomy@ucsf.edu 


\section{Abstract}

2 CRISPR systems are prokaryotic adaptive immune systems that use RNA-guided Cas nucleases

3 to recognize and destroy foreign genetic elements, like bacteriophages and plasmids. To

4 overcome CRISPR immunity, phages have evolved diverse families of anti-CRISPR proteins

5 (Acrs), each of which inhibits the nucleic acid binding or cleavage activities of specific Cas protein

6 families. Recently, Lin et al. (2020) described the discovery and characterization of 7 different Acr

7 families (AcrVIA1-7) that inhibit type VI-A CRISPR systems, which use the nuclease Cas13a to

8 perform RNA-guided RNA cleavage. In this Matters Arising article, we detail several

9 inconsistencies that question the results reported in the Lin et al. (2020) study. These include

10 inaccurate bioinformatics analyses, as well as reported experiments involving bacterial strains

11 that are impossible to construct. The authors were unable to provide their published strains with

12 which we might reproduce their experiments. We independently tested the Acr sequences

13 described in Lin et al. (2020) in two different Cas13 inhibition assays, but could not detect anti-

14 CRISPR activity. Taken together, our data and analyses prompt us to question the claim that

15 AcrVIA1-7 reported in Lin et al. are bona fide type VI anti-CRISPR proteins. 


\section{Introduction}

CRISPR-Cas systems provide their prokaryotic hosts with sequence-specific immunity against invading genetic elements, including bacteriophages and plasmids. To achieve immunity, short nucleotide sequences (spacers) are captured from foreign genomes and stored in the CRISPR locus, where they are transcribed and processed into small CRISPR RNAs (crRNAs) that guide the recognition and cleavage of elements matching the spacer. CRISPR systems are extremely diverse, and classified into 6 distinct types and 33 subtypes with different sequence content and mechanisms of interference (Makarova et al., 2020). Type VI CRISPR systems cleave RNA, using the RNA-guided RNase Cas13 to recognize phage mRNA transcripts.

To overcome immunity, many phages encode small anti-CRISPR (Acr) proteins, which use different mechanisms to inhibit the binding and/or enzymatic activities of Cas proteins. In June 2020, Wu and colleagues (Lin et al. 2020) reported the discovery of seven type VI-A antiCRISPRs (AcrVIA1-AcrVIA7) encoded by strains of Leptotrichia and Rhodobacter that inhibit Cas13a from Leptotrichia wadei (LwaCas13a), Leptotrichia shahii (LshCas13a), and Leptotrichia buccalis (LbuCas13a). The proteins were identified bioinformatically in prophages and tested with a series of assays including in vitro transcription-translation ( $\mathrm{Tx}-\mathrm{TI})$, phage and plasmid targeting assays. The authors demonstrated that these Acr proteins strongly inhibited Cas13a-mediated RNA knockdown and RNA editing in human cell lines. Separately, we (A.J.M) reported that an unrelated type VI Acr (called AcrVIA1 $1_{\text {Lse }}$ ) from a prophage of Listeria seeligeri, inhibits Cas13a from Listeria seeligeri (Meeske et al., 2020).

While reviewing the Lin et al. 2020 study, we discovered numerous troubling issues with their approach and results. Here we summarize our concerns: (i) there is no bioinformatic evidence supporting the conclusion that AcrVIA1-7 from Lin et al. are anti-CRISPR proteins, (ii) several strains reported as being used for experiments in the paper are not possible to construct 
41 due to plasmid incompatibility, (iii) none of the 7 proteins tested had anti-CRISPR activity against

42 LwaCas13a or LbuCas13a in our own bacterial assays, and (iv) the two most potent reported

43 proteins, AcrVIA4 and AcrVIA5, did not substantially inhibit LwaCas13a-mediated RNA

44 knockdown in human cells.

\section{Results}

Lin et al. used bioinformatic strategies to identify type VI Acr candidates. Specifically, they searched public sequence databases for bacterial genomes that have type VI CRISPR-Cas loci with spacers targeting another region in the same genome (often referred to as "self-targeting spacers"). A spacer and its target should not be able to stably coexist in the same cell, therefore

51 the presence of self-targeting spacers suggests that the CRISPR system might be inhibited by an anti-CRISPR protein encoded by a prophage residing in the same genome (Rauch et al., 2017).

53 This approach has been successfully used to identify anti-CRISPRs for DNA-targeting type II and

54 type V CRISPR systems (Marino et al., 2018; Rauch et al., 2017; Watters et al., 2018), but not

55 previously applied for Type VI systems, which require a transcribed target in the correct 56 orientation for basepairing (Abudayyeh et al., 2016; Meeske et al., 2019). Lin et al. identified a

57 single strain of Leptotrichia wadei (str. F0279) that possessed three self-targeting spacers

58 associated with its type VI-A CRISPR system. Next, the authors identified three prophage regions

59 in the L. wadei F0279 genome and looked for candidate anti-CRISPR genes within them. To

60 narrow down the list of phage genes, the authors claim to have identified three anti-CRISPR-

61 associated (aca) genes in the prophages (1 per prophage) and examined genes near the aca

62 genes for anti-CRISPR activity. Aca proteins are so-named based on their genomic position being

63 adjacent to known acr genes (Bondy-Denomy et al., 2013; Pawluk et al., 2014, 2016a, 2016b;

64 Pinilla-Redondo et al., 2020). Mechanistically, Aca proteins are helix-turn-helix (HTH) motif 

et al., 2019). In Fig. 1D of the original report, the authors incorrectly label the genes encoding for proteins with HTH-motifs as "aca1, aca2, and aca3" which match the names of aca genes previously used for acr discovery. Adjacent to these "aca" genes, the authors identified 5 putative type VI Acrs (acrVIA1-5) in the L. wadei F0279 genome, the most acrs reported in a single genome to date. The "aca" genes identified do encode proteins with HTH motifs, common to many phage proteins, but are not homologous to Aca1, Aca2, or Aca3, and are therefore not aca genes a priori. Thus, there is no bioinformatic basis to designate these genes acas, and no basis to suggest the genes located nearby are acrs. The ORF depictions in Fig. 1D also mask the true

74 size of the genes and the spacing between them (Fig. 1). The organization of the operons and 75 size of the Aca proteins also does not resemble other acr-aca operons. For example, "aca1" and 76 "aca2" are larger than previously identify aca genes, and in the case of "aca2," it appears to be 77 the first gene in an adjacent operon, and not co-encoded with the identified acrVIA3, acrVIA4.

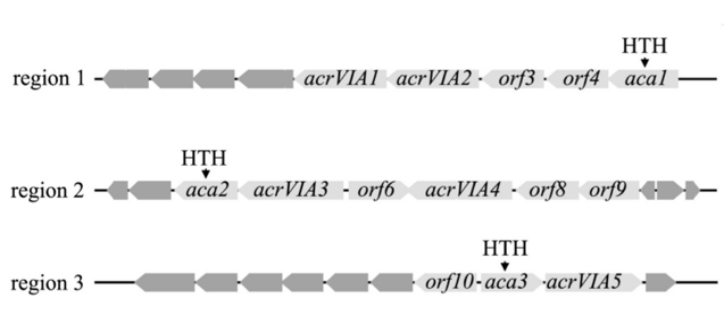

Figure 1D from Lin et al.

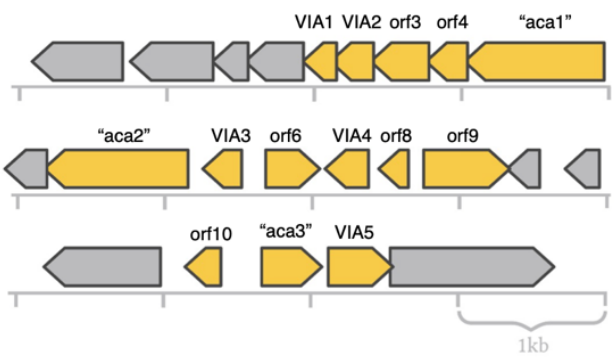

Redrawn orf maps to scale

Figure 1: Gene organization of reported Acrs. Misnamed aca genes and adjacent acr genes as depicted in Lin et al. 2020 (left) and with ORFs redrawn to reflect their true scale and spacing (right).

\section{8}

Two additional type VI acrs (acrVIA6, acrVIA7) were identified by looking at genes adjacent to homologs of known acr genes harbored by bacterial strains also possessing type $\mathrm{VI}$ CRISPR loci. The discovery of acrVIA6 was based on a nearby gene described as a homolog of the previously discovered acrlC1 (Fig. 2A). However, the gene schematized acrlC1 is 
83 misinterpreted as having $34.8 \%$ identity to the

84 originally published acrlC1 (Marino et al., 2018).

85 Using BLASTp with default parameters against

86 the nr database does not present this protein,

87 which has few identifiable homologs. However,

88 we were able to recreate that $34.8 \%$ identity

89 using pairwise BLASTp that calculated $8 / 23$

90 identical residues (34.78\%) but to only $12 \%$ of

91 the query protein $(E-v a l u e=0.002)($ Fig. 2B $)$.

92 Similarly, the authors claimed to identify acrllA1

93 and acrllC4 homologs in Leptotrichia buccalis

94 str. DSM1135, which led them to acrVIA7.

95 Again, using the AcrllC4 sequence (Lee et al.,

96 2018) for BLASTp against the $\mathrm{nr}$ database does

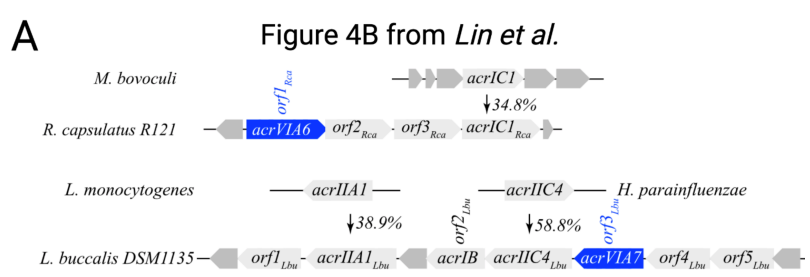

AcrIC1 BLASTp results
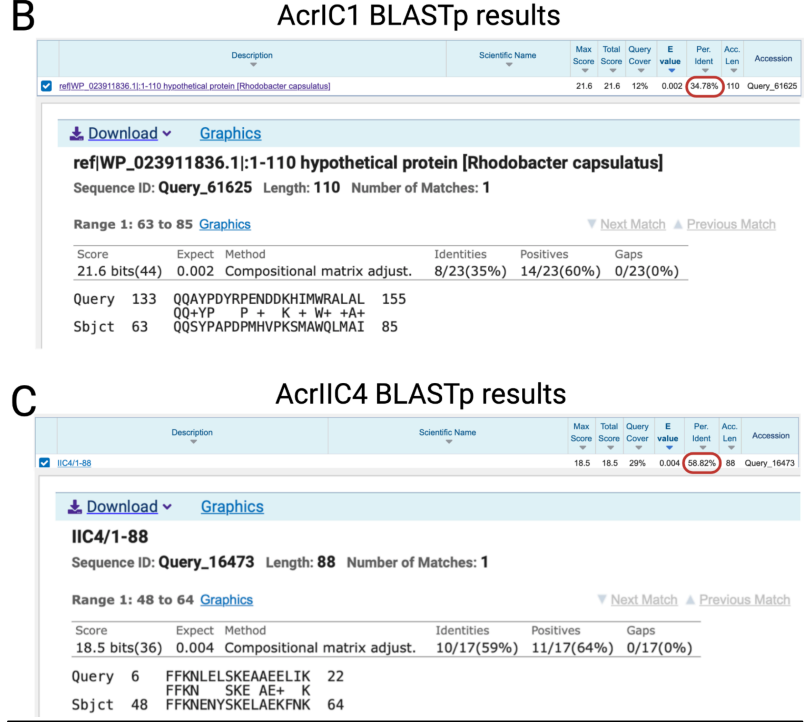

Figure 2: Misidentification of Acr homologs. (A) Reported Acr homologs with percent identity for each match indicated in Lin et al. 2020. BLASTp alignments demonstrating no significant homologs are present in the target genomes for (B) AcrlC1 or (C) AcrllC4.

97 not present homologs in this isolate. But again we were able to recreate the erroneous identity 98 value of $58.8 \%$ using pairwise BLASTp (10/17 residues align, over $29 \%$ of the protein) (Fig. 2C).

99 We could not recreate the exact 38.9\% identity of AcrlIA1 (Rauch et al., 2017) with the homolog 100 they identify. It is likely another erroneous value generated by pairwise BLASTp.

Notably, we struggled to recreate these alignments, and the authors provided no alignments of the homologs to demonstrate the percent identities. Similarly, no guidance was 103 provided towards how BLAST searches were conducted, what the parameters were, or if PSIBLAST was used. We conclude that every acr gene candidate was identified under unusual presuppositions, either through the selection of random $\mathrm{HTH}$-containing proteins in prophages 
aca1, aca2, aca3), or through the identification of incorrect homologs of known acr genes

108

109

(acrVIA6-7 and acrIB1 in Fig. 4B in the original paper).

Despite the lack of bioinformatic evidence, it remained possible that the seven candidate proteins identified by Lin et al. were indeed Acr proteins, as demonstrated through multiple experimental approaches in the original report. To test them experimentally, we first sought to reproduce some of the findings from the original study using the authors' published reagents. All of the Acr proteins were shown by Lin et al. to reverse Cas13a-mediated interference against the E. coli RNA bacteriophage MS2. In hopes of repeating this experiment, we requested the published bacterial strains harboring LwaCas13a, an MS2-targeting guide, each of the individual Acrs, and an additional no-Acr control. The corresponding author did not cooperate with our request, and seven months later informed us that he could not locate the published strains. Some strains were eventually sent, however, they were not the ones we requested.

We then planned to reconstruct the strains ourselves with plasmids that were provided, but immediately realized that the strains cannot be constructed as described in the original study. MS2 phage infects the $E$. coli strain $\mathrm{C} 3000$, and each of the published strains contains three plasmids: (i) either an LwaCas13a or LbuCas13a expression plasmid in the vector backbone p2CT (these were generated by the Doudna lab and deposited in Addgene); (ii) an MS2-targeting guide RNA plasmid in vector backbone pCDFDuet; and (iii) an Acr expression plasmid in vector backbone pET16b. There are two major problems with these plasmid combinations. Most importantly, the p2CT backbone (East-Seletsky et al., 2016) and pET16b backbone are incompatible plasmids containing the same antibiotic resistance marker and the same origin of replication. Therefore, these strains are impossible to construct. Second, both of these backbones are designed for T7 RNA polymerase-driven gene expression for protein purification and are normally used in DE3-lysogenized host strains containing a copy of T7 RNAP (Studier et al., 
1990). Strain C3000, a K12 derivative, has no copy of T7 RNAP, and therefore would drive expression of neither Cas13a nor the Acr.

Another method that Lin et al used to demonstrate Acr activity was a plasmid

transformation assay in E. coli. Cas13a restricts the transformation of plasmids expressing target

RNA, but AcrVIA1-5 were shown to relieve this restriction. Again, we encountered difficulty when

trying to repeat the experiments as performed in the original study. The experiment described by

However, such a self-targeting Cas13 plasmid should not be possible to construct in the first place, unless cas13a or the gentamicin resistance gene were conditionally expressed. The paper contains no information about how any of the plasmids were constructed, and there is no

144 description of an inducible promoter used in this plasmid. We requested the strain carrying this 145 plasmid from the corresponding author, and although it was listed among the shipped strains, we 146 were unable to culture it in the specified antibiotics.

148 performed plasmid transformation assays in E. coli. We designed plasmids carrying either 149 LbuCas13a or LwaCas13a crRNAs targeting the kanamycin resistance marker of a second plasmid (Fig. 3A). To test Cas13a function, we first transformed strains harboring each Cas13a

151 plasmid (or empty vector) with the kanR plasmid. As expected, we found that both Cas $13 a$ 152 homologs prevented transformation with the targeted plasmid in a crRNA-dependent manner 153 (Fig. 3B, no Acr control). To test the putative Acrs, we synthesized codon-optimized alleles of 
promoter (Fig. 3A). In contrast to the robust Cas13a inhibition reported by Lin et al, we found that none of the seven proteins reduced Cas13 targeting (Fig. 3B). As a positive control to ensure AcrVIA1 $1_{\text {Lse }}$ target plasmid was completely resistant to LseCas13a. All of the Acrs in this

A

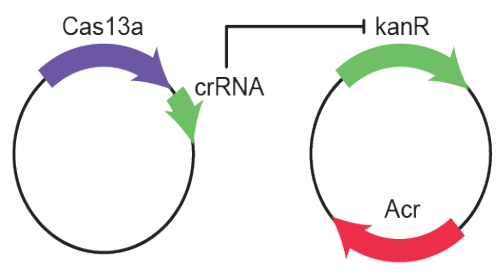

Cas13 targeting:

plasmids cannot coexist

Cas13 inhibited: plasmids can coexist

\section{B}
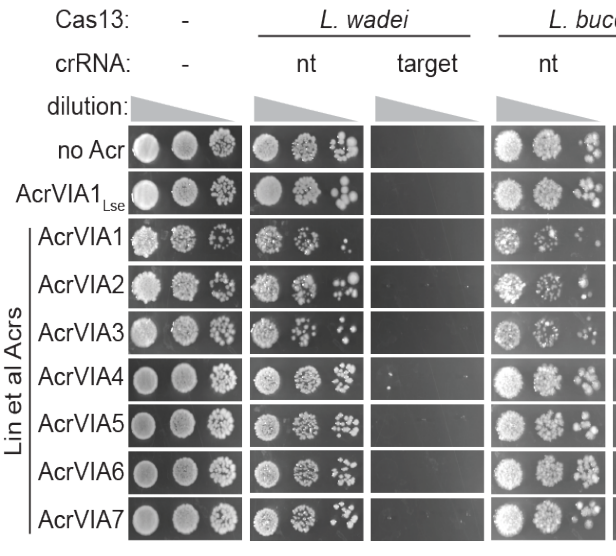

Figure 3: Target plasmid transformation assay to measure Acr function. (A) Assay schematic. E. coli strains carried plasmids with Cas13a from $L$. wadei, L. buccalis, or $L$. seeligeri and a crRNA targeting the kanamycin resistance marker of a second plasmid (or non-targeting crRNA control), which also candidate Acr proteins. Cas 13a targeting of the kanR marker restricts transformation of the target plasmid, unless the Acr inhibits Cas13a. (B) Results of transformation assay. The indicated recipient strains (columns) were transformed with the indicated plasmids (rows), serially diluted and spotted onto media selecting for both plasmids. nt, non-targeting crRNA. 
Next, we sought to recapitulate the author's demonstration of potent inhibition of

173 LwaCas13a in human cells with their putative Acr proteins. Lin et al. report near-complete

174 inhibition of LwaCas13a transcript degradation by AcrVIA5 and high levels of inhibition for several

175 other candidate AcrVIAs. We performed experiments in HEK 293T cells with LwaCas13a and the

176 Lin et al. crRNAs targeting endogenous KRAS and PPIB transcripts (Figs. 4A and 4B,

177 respectively), in the absence or presence of varying amounts of plasmids encoding their two most

178 potent reported Acrs, AcrVIA5 and AcrVIA4. As negative controls, we also examined AcrlIA4 and

179 AcrllA5, which inhibit Cas9 (Fig. 4). Across the various quantities of Acr plasmids that we

180 examined, RT-qPCR analysis at 48-hours post-transfection revealed largely inconsequential

181 reduction of transcript degradation by AcrVIA5 or AcrVIA4; inhibition was not meaningfully

182 different than what we observed in the presence of AcrlIA4 or AcrlIA5, which are not expected to 183 interact with LwaCas13a. In our experiments, the highest doses of AcrVIA4 or AcrVIA5 expression

184 plasmid were a 4.32x excess molar ratio relative to the LwaCas $13 a$ expression plasmid, which is

185 comparable to the approximately $5.7 x$ excess reported by Lin et al. For context, using similar

186 construct architectures (plasmid backbones, promoters, codon usage, etc.) we previously

187 demonstrated that AcrlIA4 and AcrlIA5 exhibit robust inhibition of SpCas9 at AcrllA:Cas9 plasmid

188 molar ratios as low as 0.1 (Mahendra et al., 2020; Osuna et al., 2020b). Together, our results do

189 not support the conclusion from Lin et al. that AcrVIA5 and AcrVIA4 strongly inhibit LwaCas13a

190 activity in human cells. 
A

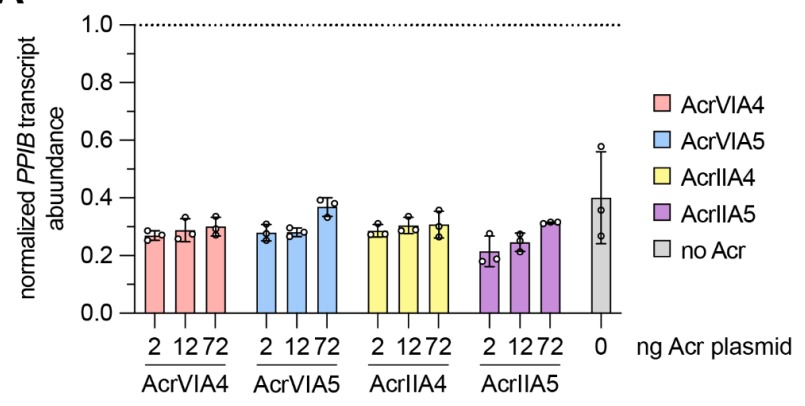

B

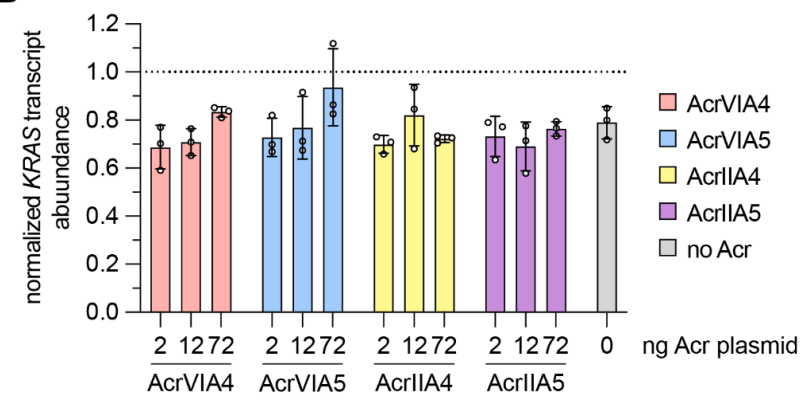

Figure 4: Assessment of AcrVIA-mediated inhibition of LwaCas13a. (A, B) Knockdown of endogenous PPIB (panel A) or KRAS (panel B) transcripts by LwaCas13a in the presence of various amounts of Acr expression plasmids was determined via RT-qPCR analysis $(n=3$ biological replicates; dots represent the mean of three technical triplicate qPCR values with SD shown). PPIB or KRAS RNA levels were normalized to ACTB and knockdown was determined by comparison to a non-targeting LwaCas13a control. Increasing amounts of plasmids encoding the anti-CRISPR proteins AcrVIA5, AcrVIA4, AcrlIA4, and AcrlIA5 were added to a consistent amount of LwaCas13a nuclease and gRNA plasmids. The molar ratios of the Acr to LwaCas13a expression plasmids were approximately $0.12,0.72$, and 4.32 for the $2 \mathrm{ng}, 12 \mathrm{ng}$, and $72 \mathrm{ng}$ treatments, respectively.

Here, we have presented multiple lines of evidence to challenge the findings put forth by Lin et al. (2020) pertaining to the discovery of seven Type VI-A Acr proteins. Bioinformatic inconsistencies are detailed, along with plasmid incompatibilities and independent experiments that could not establish the functionality of these proteins. Given the interest and utility envisioned for Acr proteins, we hope that this report is of use for the field and welcome any feedback supporting or refuting our claims. 
bioRxiv preprint doi: https://doi.org/10.1101/2021.05.27.445852; this version posted May 27, 2021. The copyright holder for this preprint (which

was not certified by peer review) is the author/funder, who has granted bioRxiv a license to display the preprint in perpetuity. It is made available under aCC-BY-NC 4.0 International license.

\section{Acknowledgements}

204 This work in the Bondy-Denomy lab (J.B.-D). is supported by the UCSF Program for Breakthrough 205 Biomedical Research funded in part by the Sandler Foundation, the NIH [DP5-OD021344, R01GM127489], 206 and DARPA HR0011-17-2-0043. M.J. is supported by the NSF GRFP Fellowship. B.P.K. acknowledges 207 support from NIH P01-HL142494 and the Margaret Q. Landenberger Research Foundation. A.J.M was a 208 Helen Hay Whitney postdoctoral fellow.

Conflict of interest statement. J.B.-D. is a scientific advisory board member of SNIPR Biome and Excision 211 Biotherapeutics and a scientific advisory board member and co-founder of Acrigen Biosciences. J.B.-D. is 212 an inventor on patents filed by UCSF pertaining to anti-CRISPR technology. B.P.K is an inventor on patents 213 and patent applications filed by Mass General Brigham that describe genome engineering technologies. 214 B.P.K. consults for Avectas Inc., EcoR1 capital, and ElevateBio, and is an advisor to Acrigen Biosciences and Life Edit Therapeutics. 


\section{References}

219 Abudayyeh, O.O., Gootenberg, J.S., Konermann, S., Joung, J., Slaymaker, I.M., Cox, D.B.T., 220 Shmakov, S., Makarova, K.S., Semenova, E., Minakhin, L., et al. (2016). C2c2 is a single221 component programmable RNA-guided RNA-targeting CRISPR effector. Science 353, aaf5573.

222 Birkholz, N., Fagerlund, R.D., Smith, L.M., Jackson, S.A., and Fineran, P.C. (2019). The 223 autoregulator Aca2 mediates anti-CRISPR repression. Nucleic Acids Res. 47, 9658-9665.

224 Bondy-Denomy, J., Pawluk, A., Maxwell, K.L., and Davidson, A.R. (2013). Bacteriophage genes 225 that inactivate the CRISPR/Cas bacterial immune system. Nature 493, 429-432.

226 East-Seletsky, A., O'Connell, M.R., Knight, S.C., Burstein, D., Cate, J.H.D., Tjian, R., and Doudna, J.A. (2016). Two distinct RNase activities of CRISPR-C2c2 enable guide-RNA processing and RNA detection. Nature 538, 270-273.

Lee, J., Mir, A., Edraki, A., Garcia, B., Amrani, N., Lou, H.E., Gainetdinov, I., Pawluk, A., Ibraheim, R., Gao, X.D., et al. (2018). Potent Cas9 Inhibition in Bacterial and Human Cells by AcrlIC4 and AcrlIC5 Anti-CRISPR Proteins. MBio 9.

232 Lin, P., Qin, S., Pu, Q., Wang, Z., Wu, Q., Gao, P., Schettler, J., Guo, K., Li, R., Li, G., et al. 233 (2020). CRISPR-Cas13 Inhibitors Block RNA Editing in Bacteria and Mammalian Cells. Mol. 234 Cell 78, 850-861.e5.

Mahendra, C., Christie, K.A., Osuna, B.A., Pinilla-Redondo, R., Kleinstiver, B.P., and BondyDenomy, J. (2020). Broad-spectrum anti-CRISPR proteins facilitate horizontal gene transfer. Nat Microbiol 5, 620-629.

Makarova, K.S., Wolf, Y.I., Iranzo, J., Shmakov, S.A., Alkhnbashi, O.S., Brouns, S.J.J.,

Marino, N.D., Zhang, J.Y., Borges, A.L., Sousa, A.A., Leon, L.M., Rauch, B.J., Walton, R.T., V CRISPR-Cas inhibitors. Science 362, 240-242.

Meeske, A.J., Nakandakari-Higa, S., and Marraffini, L.A. (2019). Cas13-induced cellular dormancy prevents the rise of CRISPR-resistant bacteriophage. Nature 570, 241-245. Marraffini, L.A. (2020). A phage-encoded anti-CRISPR enables complete evasion of type VI-A CRISPR-Cas immunity. Science.

Osuna, B.A., Karambelkar, S., Mahendra, C., Sarbach, A., Johnson, M.C., Kilcher, S., and Bondy-Denomy, J. (2020a). Critical Anti-CRISPR Locus Repression by a Bi-functional Cas9 Inhibitor. Cell Host Microbe 28, 23-30.e5.

252 Osuna, B.A., Karambelkar, S., Mahendra, C., Christie, K.A., Garcia, B., Davidson, A.R., 253 Kleinstiver, B.P., Kilcher, S., and Bondy-Denomy, J. (2020b). Listeria Phages Induce Cas9 254 Degradation to Protect Lysogenic Genomes. Cell Host Microbe 28, 31-40.e9. 
Pawluk, A., Bondy-Denomy, J., Cheung, V.H.W., Maxwell, K.L., and Davidson, A.R. (2014). A new group of phage anti-CRISPR genes inhibits the type I-E CRISPR-Cas system of Pseudomonas aeruginosa. MBio 5, e00896.

Pawluk, A., Staals, R.H.J., Taylor, C., Watson, B.N.J., Saha, S., Fineran, P.C., Maxwell, K.L., and Davidson, A.R. (2016a). Inactivation of CRISPR-Cas systems by anti-CRISPR proteins in diverse bacterial species. Nature Microbiology 1,1-6.

Pawluk, A., Amrani, N., Zhang, Y., Garcia, B., Hidalgo-Reyes, Y., Lee, J., Edraki, A., Shah, M., Sontheimer, E.J., Maxwell, K.L., et al. (2016b). Naturally Occurring Off-Switches for CRISPRCas9. Cell 167, 1829-1838.e9.

Pinilla-Redondo, R., Shehreen, S., Marino, N.D., Fagerlund, R.D., Brown, C.M., Sørensen, S.J., Fineran, P.C., and Bondy-Denomy, J. (2020). Discovery of multiple anti-CRISPRs highlights anti-defense gene clustering in mobile genetic elements. Nat. Commun. 11, 5652.

Rauch, B.J., Silvis, M.R., Hultquist, J.F., Waters, C.S., McGregor, M.J., Krogan, N.J., and Bondy-Denomy, J. (2017). Inhibition of CRISPR-Cas9 with Bacteriophage Proteins. Cell 168, 150-158.e10.

Stanley, S.Y., Borges, A.L., Chen, K.-H., Swaney, D.L., Krogan, N.J., Bondy-Denomy, J., and Studier, F.W., Rosenberg, A.H., Dunn, J.J., Dubendorff, J.W. (1990). Use of T7 RNA polymerase to direct expression of cloned genes. Methods Enzymol. 185:60-89 CRISPR Transcription. Cell 178, 1452-1464.e13. 


\section{Methods}

\section{Bacterial strains and culture conditions}

281 Plasmid culture and extraction, as well as plasmid transformation assays were performed with 282 New England Biolabs Turbo Competent E. coli. Bacteria were grown in Lysogeny Broth (LB) 283 supplemented with antibiotics: kanamycin $(50 \mu \mathrm{g} / \mathrm{mL})$, chloramphenicol $(25 \mu \mathrm{g} / \mathrm{ml})$, and 284 anhydrotetracyline $(100 \mathrm{ng} / \mathrm{mL}) .0 .2 \%$ arabinose was added to induce expression of Cas 13 from 285 Para promoters.

\section{Plasmid construction}

287 Cas13 plasmids for transformation assay were cloned into the vector pAM38 containing p15A 288 origin of replication, chloramphenicol resistance marker, and an arabinose-inducible Para 289 promoter. Coding sequences for LwaCas13a, LbuCas13a, or LseCas13a were inserted 290 downstream of the Para promoter, and crRNA constructs were inserted downstream of the cas13 291 coding sequence driven by a synthetic promoter (J23119). These plasmids were constructed by 292 three piece Gibson assembly as described below and validated by Sanger sequencing. Lists of 293 plasmids, oligonucleotide primers, and crRNAs can be found in Supplementary Tables.

294 LwaCas13a plasmids: Three piece Gibson assembly using (1) Sall/HindIII-digested pAM38, (2) 295 LwaCas13a coding sequence and Shine-Dalgarno sequence amplified from Addgene \#91865 296 using primers oAM1496 and oAM1497, and (3) J23119-driven crRNA gBlock fragments 297 synthesized by IDT was used to generate pAM491 (LwaCas13a kan crRNA) and pAM494 298 (LwaCas13a non-targeting crRNA).

LbuCas13a plasmids: Three piece Gibson assembly using (1) Sall/HindIII-digested pAM38, (2) LbuCas13a coding sequence codon-optimized for E. coli (synthesized by Genewiz) and ShineDalgarno sequence amplified using primers oAM211 and oAM1477, and (3) J23119-driven crRNA gBlock fragments synthesized by IDT was used to generate pAM492 (LbuCas13a kan crRNA) and pAM475 (LbuCas13a non-targeting crRNA).

LseCas13a plasmids: Three piece Gibson assembly using (1) Sall/HindIII-digested pAM38, (2) LseCas13a coding sequence codon-optimized for E. coli (synthesized by Genewiz) and ShineDalgarno sequence amplified using primers oAM207 and oAM994, and (3) J23119-driven crRNA gBlock fragments synthesized by IDT was used to generate pAM526 (LseCas13a kan crRNA) and pAM319 (LseCas13a non-targeting crRNA).

Acr plasmids: Acrs were expressed from pAM326 (carrying kanamycin resistance marker and pWV01 origin of replication) and driven by an anhydrotetracycline-inducible Ptet promoter. Acr coding sequences were synthesized by Genewiz, amplified using primers oAM1529 and oAM1530, and inserted with Ptet fragment into HindlII/Eagl-digested pAM326 via three piece Gibson assembly to make pAM495 (AcrVIA1), pAM496 (AcrVIA2), pAM497 (AcrVIA3), pAM498 
(AcrVIA4), pAM499 (AcrVIA5), pAM500 (AcrVIA6), pAM501 (AcrVIA7). The AcrVIA1Lse plasmid pAM383 was generated previously (Meeske et al. 2020).

\section{Plasmid transformation assay}

317 NEB Turbo competent E. coli were transformed with either empty vector or plasmids harboring 318 LwaCas13a, LbuCas13a, or LseCas13a and expressing non-targeting or targeting crRNAs, and 319 selected on LB agar containing chloramphenicol and $0.2 \%$ glucose to repress Cas 13 a expression. 320 Each transformed strain was made chemically competent by resuspension of exponentially 321 growing cells in $1 / 5$ culture volume of ice cold TFB I $(10 \mathrm{mM} \mathrm{CaCl} 2,30 \mathrm{mM}$ potassium acetate, $\mathrm{pH}$ $3225.8,100 \mathrm{mM} \mathrm{RbCl}, 50 \mathrm{mM} \mathrm{MnCl} 2,15 \%$ glycerol), incubation on ice for 15 minutes, then pelleting 323 and resuspension in 1/25 culture volume of ice cold TFB II (10 mM MOPS pH 6.5, $10 \mathrm{mM} \mathrm{RbCl}$, $75 \mathrm{mM} \mathrm{CaCl} 2,15 \%$ glycerol). Chemically competent cells were transformed by heat shock at $42^{\circ} \mathrm{C}$ with $100 \mathrm{ng}$ Acr plasmids or empty vector control, then recovered for 1 hour in LB. Tenfold serial dilutions of recovered transformants were made and $5 \mu \mathrm{L}$ of each dilution was spotted onto LB agar supplemented with kanamycin, chloramphenicol, arabinose, and anhydrotetracycline. Plates were photographed after 1 day of incubation at $37^{\circ} \mathrm{C}$.

\section{Human cell culture and transfections}

Human HEK 293T cells (ATCC) were cultured in Dulbecco's Modified Eagle Medium (DMEM) supplemented with 10\% heat-inactivated FBS (HI-FBS) and $1 \%$ penicillin/streptomycin. Supernatants from cell cultures were tested monthly for mycoplasma using MycoAlert PLUS (Lonza). For transfections, approximately $2 \times 10^{4}$ cells per well were seeded in 96-well plates. Approximately 18-22 hours after seeding, transfections were performed using TransIT-X2 (Mirus) transfection reagent with $60 \mathrm{ng}$ of LwaCas13a plasmid (Addgene ID 91924), $20 \mathrm{ng}$ of gRNA plasmid (generated by cloning oligos into an LwaCas13a crRNA entry plasmid, LTH151, Addgene ID 171129), and with 0, 2, 12, or $72 \mathrm{ng}$ of Acr plasmid. The human codon optimized Acr constructs for AcrVIA4 and AcrVIA5 were synthesized by Twist Biosciences and cloned into a pCMV backbone (plasmid IDs LTH956 and LTH957 respectively; see Supplementary Sequences), similar to how we previously generated the human cell expression constructs for AcrllA4 and AcrlIA5 (Addgene IDs 133801 and 133802, respectively) (see Supplementary Sequences). The total amount of DNA in each transfection remained constant (152 ng), with a pCMV-null plasmid (Addgene ID 171128) used to balance DNA amounts when titrating the Acr plasmid. The plasmid mixtures were combined with $0.54 \mathrm{ul}$ of TransIT-X2 and volume of Opti-MEM (ThermoFisher) to a final volume of $15 \mathrm{ul}$, incubated at room temperature for 15 minutes, and then applied to the

\section{RNA extraction and RT-qPCR}

At 48 hours post-transfection, total RNA was extracted from transfected cells using an RNeasy Plus Mini Kit (Qiagen; cat. no. 74136). Purified RNA was reverse transcribed using a HighCapacity RNA-to-cDNA kit (ThermoFisher; cat. no. 4388950) using up to 250 ng of RNA as input. 
prepared in technical triplicate with 5 ul of Fast SYBR Green Master Mix (ThermoFisher; cat. no. 4385610), 3 ul diluted cDNA, and 2 ul qPCR primer pairs specific to the target (IDT; Supplementary Table 1). Control reactions amplifying ACTB were set up in parallel for each cDNA library. Reaction cycling was performed using a Roche LightCycler480. To determine the fold change of each sample, the expression levels were normalized to a negative control transfection containing LwaCas13a plasmid and an empty pUC19 backbone plasmid (Addgene ID 133961). Each qPCR reaction was analyzed for purity by melting curve analysis to confirm a 359 single PCR product.

Supplementary Table 1. Sequences of gRNAs and oligonucleotides

\begin{tabular}{|l|l|}
\hline Description & Nucleotide Sequence \\
\hline gRNA spacer, KRAS & AATTTCTCGAACTAATGTATAGAAGGCA \\
\hline gRNA spacer, PPIB & TCCTTGATTACACGATGGAATTTGCTGT \\
\hline qPCR Forward primer, KRAS & CAAGAGTGCCTTGACGATACA \\
\hline qPCR Reverse primer, KRAS & GACCTGCTGTGTCGAGAATATC \\
\hline qPCR Reverse Primer, PPIB & GCTCACCGTAGATGCTCTTT \\
\hline qPCR Forward primer, PPIB & GGAGAGAAAGGATTTGGCTACA \\
& \\
\hline
\end{tabular}




\begin{tabular}{|l|l|}
\hline qPCR Reverse primer, ACTB & AGGTCTTTGCGGATGTCCACGT \\
\hline Lwa non-targeting crRNA & $\begin{array}{l}\text { gatttagactacccaaaaacgaaggggactaaaacG } \\
\text { TGCCCAGTCATAGCCGAATAGCCTCTC } \\
\text { CA }\end{array}$ \\
\hline Lwa kan crRNA & $\begin{array}{l}\text { gatttagactaccccaaaaacgaaggggactaaaacT } \\
\text { ATTTTTCGATCAGTTTTTCAATTCCGG } \\
\text { T }\end{array}$ \\
\hline Lbu non-targeting crRNA & $\begin{array}{l}\text { gatttagaccaccccaaaaatgaaggggactaaaacG } \\
\text { TGCCCAGTCATAGCCGAATAGCCTCTC } \\
\text { CA }\end{array}$ \\
\hline Lbu kan crRNA & $\begin{array}{l}\text { gatttagaccaccccaaaaatgaaggggactaaaacT } \\
\text { ATTTTTCGATCAGTTTTTCAATTCCG } \\
\text { T }\end{array}$ \\
\hline Lse non-targeting crRNA & $\begin{array}{l}\text { gtaagagactacctctatatgaaagaggactaaaacGT } \\
\text { GCCCAGTCATAGCCGAATAGCCTCTCC } \\
\text { A }\end{array}$ \\
\hline Lse kan crRNA & $\begin{array}{l}\text { gtaagagactacctctatatgaaagaggactaaaacTA } \\
\text { GCAGGAGACATTCCTTCCGTATCTTTTA }\end{array}$ \\
\hline
\end{tabular}

362 Supplementary Sequences

363 Amino acid and nucleotide sequences for constructs used in human cell experiments. Linker in 364 red. NLS in green.

365 >pCMV-T7-AcrVIA4 (LTH956)

366 MDKANRCLKAKDKILNILEKEEITLDEFNNISKDIAKEYVEKAVLKPKDIAERIINMVKNAKSISFDE 367 LASEISEE 
ATGGACAAGGCCAACAGATGCCTGAAGGCCAAGGACAAGATCCTGAACATCCTGGAGAAG GAGGAGATCACCCTGGACGAGTTCAACAACATCAGCAAGGACATCGCCAAGGAGTACGTG GAGAAGGCCGTGCTGAAGCCCAAGGACATCGCCGAGAGAATCATCAACATGGTGAAGAAC GCCAAGAGCATCAGCTTCGACGAGCTGGCCAGCGAGATCAGCGAGGAG

373 MERNFKKVTENTGRKEVFKVMHDKVEIINDFNTNEKREARIIFHDQKIYVILYQNLNFEELKWLN 374 FYILIYGNQSYGKNTFFEFKLNKNNLIYHLQVWNIIENKKFKSKSISLLVKALSSKAGV

ATGGAGAGAAACTTCAAGAAGGTGACCGAGAACACCGGCAGAAAGGAGGTGTTCAAGGTG ATGCACGACAAGGTGGAGATCATCAACGACTTCAACACCAACGAGAAGAGAGAGGCCAGA ATCATCTTCCACGACCAGAAGATCTACGTGATCCTGTACCAGAACCTGAACTTCGAGGAGC TGAAGTGGCTGAACTTCTACATCCTGATCTACGGCAACCAGAGCTACGGCAAGAACACCTT CTTCGAGTTCAAGCTGAACAAGAACAACCTGATCTACCACCTGCAGGTGTGGAACATCATC GAGAACAAGAAGTTCAAGAGCAAGAGCATCAGCCTGCTGGTGAAGGCCCTGAGCAGCAAG

MGNINDLIREIKNKDYTVKLSGTDSNSITQLIIRVNNDGNEYVISESENESIVEKFISAFKNGWNQ EYEDEEEFYNDMQTITLKSELNGSGGGGSGPKKKRKV

ATGGGCAACATCAACGACCTGATCAGAGAGATCAAGAACAAGGACTACACCGTGAAGCTG AGCGGCACCGACAGCAACAGCATCACCCAGCTGATCATCAGAGTGAACAACGACGGCAAC GAGTACGTGATCAGCGAGAGCGAGAACGAGAGCATCGTGGAGAAGTTCATCAGCGCCTTC AAGAACGGCTGGAACCAGGAGTACGAGGACGAGGAGGAGTTCTACAACGACATGCAGAC CATCACCCTGAAGAGCGAGCTGAACGGATCCGGCGGTGGAGGCAGTGGGCCCAAGAAGA AGAGGAAAGTC

>pCMV-T7-AcrlIA5-NLS(SV40) (KAC203; Addgene ID 133802)

MAYGKSRYNSYRKRNFSISDNQRREYAKKMKELEQAFENLDGWYLSSMKDSAYKDFGKYEIR LSNHSADNRYHDLENGRLIVNVKASKLNFVDIIENKLGKIIEKIDTLDLDKYRFINATKLERDIKCYY KGYKTKKDVIGSGGGGSGPKKKRKV

ATGGCCTACGGCAAGAGCAGATACAACAGCTACAGAAAGAGAAACTTCAGCATCAGCGAC AACCAGAGAAGAGAGTACGCCAAGAAGATGAAGGAGCTGGAGCAGGCCTTCGAGAACCT GGACGGCTGGTACCTGAGCAGCATGAAGGACAGCGCCTACAAGGACTTCGGCAAGTACG AGATCAGACTGAGCAACCACAGCGCCGACAACAGATACCACGACCTGGAGAACGGCAGAC TGATCGTGAACGTGAAGGCCAGCAAGCTGAACTTCGTGGACATCATCGAGAACAAGCTGG GCAAGATCATCGAGAAGATCGACACCCTGGACCTGGACAAGTACAGATTCATCAACGCCA CCAAGCTGGAGAGAGACATCAAGTGCTACTACAAGGGCTACAAGACCAAGAAGGACGTGA TCGGATCCGGCGGTGGAGGCAGTGGGCCCAAGAAGAAGAGGAAAGTC 
bioRxiv preprint doi: https://doi.org/10.1101/2021.05.27.445852; this version posted May 27, 2021. The copyright holder for this preprint (which

was not certified by peer review) is the author/funder, who has granted bioRxiv a license to display the preprint in perpetuity. It is made available under aCC-BY-NC 4.0 International license. 